\title{
FINAL TECHNICAL REPORT
}

PROJECT TITLE:

DOE Award Number:

Project Period:

Principal Investigator:

Recipient:

Business Contact:

Subcontractors:

Partners:

DOE Project Officer:
Gradient Index Spheres by the Sequential Accretion of Glass Powders

SBIR Phase I Award DE-FG02-07ER84737

June 20, 2007 to May 31, 2008

Mariano Velez, (573) 364-2338; mvelez@mo-sci.com

MO-SCI Corporation

4040 Hypoint Dr.,

Rolla, MO 65401

(573) 364-2338; (573) 364-9589 FAX; www.mo-sci.com

Ted Day

MO-SCl Corporation

4040 Hypoint North Dr., P.O. Box 2, Rolla, MO 65402

(573) 364-2338; (573) 364-9589 fax; tday@mo-sci.com

None

Patrick W. Mullen, 860-676-7129; patrick.mullen@reflexite.com

Reflexite Technology Center 120 Darling Dr.

Avon, CT 06001-4217

Victoria T. Franques; (202) 586-2560;

victoria.franques@nnsa.doe.gov

DOE Award Administrator: Earlette M. Robinson; (630) 252-2667;

earlette.robinson@ch.doe.gov

DOE HQ Contact: $\quad$ Sarah Dillich; (202) 586-7925; sara.dillich@ee.doe.gov

DOE Contract Specialist: Beth Dwyer; (303) 275-4719; beth.dwyer@go.doe.gov 


\section{ACKNOWLEDGEMENT, DISCLAIMER AND PROPRIETARY DATA NOTICE}

Acknowledgement: This report is based upon work supported by the U.S. Department of Energy under SBIR Phase I Award DE-FG02-07ER84737.

Disclaimer: Any findings, opinions, and conclusions or recommendations expressed in this report are those of the authors and do not necessarily reflect the views of the Department of Energy.

Proprietary Data Notice: This report contains no proprietary data or information. 


\section{EXECUTIVE SUMMARY}

The Department of Energy is seeking a method for fabricating $\mathrm{mm}$-scale spheres having a refractive index that varies smoothly and continuously from the center to its surface [1]. The fabrication procedure must allow the creation of a range of index profiles. The spheres are to be optically transparent and have a refractive index differential greater than 0.2 . The sphere materials can be either organic or inorganic and the fabrication technique must be capable of scaling to low cost production.

Mo-Sci Corporation proposed to develop optical quality gradient refractive index (GRIN) glass spheres of millimeter scale (1 to $2 \mathrm{~mm}$ diameter) by the sequential accretion and consolidation of glass powders. Other techniques were also tested to make GRIN spheres as the powder-accretion method produced non-concentric layers and poor optical quality glass spheres. Potential ways to make the GRIN spheres were (1) by "coating" glass spheres (1 to $2 \mathrm{~mm}$ diameter) with molten glass in a two step process; and (2) by coating glass spheres with polymer layers.

\section{INTRODUCTION}

Mo-Sci Corp. proposed to develop optical quality gradient refractive index (GRIN) spheres of mm-scale by the sequential accretion and subsequent melting and consolidation of glass powders onto glass spheres. The approach could allow arbitrary radial refractive index $(\mathrm{RI})$ gradients to be produced from commercially available glass powders. This approach is an adaptation of the powder pelletization method used in the mineral industry [2,3] and in the pharmaceutical industry for fabricating pellets [4]. Powder pelletization involves the conversion of fine ( 1 micron), loose powder into uniformly sized, dense, free flowing spheres by mechanical agitation.

Figure 1 illustrates the intended production of a GRIN sphere by the sequential accretion of glass powder layers. Darker shades of gray represent increasing RI. Layer $n=0$ represents a seed glass microsphere and $n=1,2,3, \ldots$ represent sequential glass powder layers of differing RI. After the desired RI gradient has been layered onto the glass microsphere seeds, the coated spheres would be consolidated in a rotary vacuum furnace or vacuum drop tube furnace to produce solid bubble free spheres. Once the spheres were consolidated, traditional polishing techniques, if needed, could be used to produce an optical quality finish on the surface of the GRIN glass spheres. 
a)
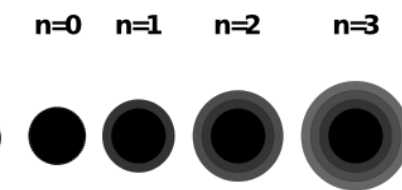

○○

$n=7$

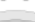

$\bullet$
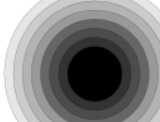

$\bullet \bullet \bullet$

b)
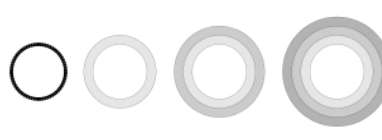

.

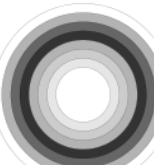

c)
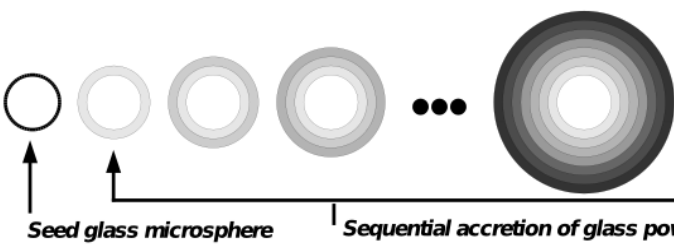

00

Figure 1. Diagram illustrating the production of a GRIN sphere by sequential accretion of glass powder layers. Darker shades represent increasing $\mathrm{RI}, \mathrm{n}=0$ is the seed glass microsphere and $n=1,2,3, \ldots$ are sequential glass powder layers of differing refractive index: a) Lundberg gradient, b) arbitrary gradient, c) inverse Lundberg gradient.

Two methods are known to produce GRIN optics of planar and cylindrical geometry from glass powders [5,6]. The first method uses different glass compositions of the desired refractive index. Schott glasses (Table 1) were used to produce GRIN rods [7]. The second method uses two glass powders with RI matching the highest and lowest values desired. Intermediate refractive indices were obtained by producing proportional mixtures of the two end-point glasses. In addition to the desired RI, the glass compositions chosen must have compatible processing properties, such as thermal expansion and melting and softening point, to facilitate consolidation to a defectfree body.

Table 1. Selected Properties of Glasses for Manufacturing GRIN Rods.

\begin{tabular}{|l|c|c|c|c|c|}
\hline $\begin{array}{l}\text { Glass } \\
\text { Code } \\
{[8]}\end{array}$ & $\begin{array}{c}\text { Refractive } \\
\text { Index, } \\
\mathrm{n}_{\mathrm{D}}\end{array}$ & $\begin{array}{c}\text { Abbe } \\
\text { Number, } \\
\mathrm{V}_{\mathrm{D}}\end{array}$ & $\begin{array}{c}\text { Thermal } \\
\text { Expansion } \\
\text { Coefficient } \\
(\mathrm{\alpha}), \mathrm{ppm} /{ }^{\circ} \mathrm{C}\end{array}$ & $\begin{array}{c}\text { Glass Transition } \\
\text { Temperature } \\
(\mathrm{Tg}),{ }^{\circ} \mathrm{C}\end{array}$ & $\begin{array}{c}\text { Density, } \\
\mathrm{g} / \mathrm{cm}^{3}\end{array}$ \\
\hline SSKN5 & 1.65844 & 50.88 & 6.8 & 641 & 3.71 \\
\hline SK15 & 1.62299 & 58.06 & 6.9 & 634 & 3.64 \\
\hline SK2 & 1.60738 & 56.65 & 6.0 & 654 & 3.55 \\
\hline
\end{tabular}




\section{RESULTS AND DISCUSSION}

The results indicate that $1-\mathrm{mm}$ diameter glass spheres can be coated with multiple glass layers of thickness between 50 and 100 microns by powder accretion and consolidated by melting as proposed. However, the surface quality of the final glass spheres is poor, showing defects such as bubbles and cracks. Four additional processes were tested which have a potential for making GRIN glass spheres: (a) spheroidizing of fibers pulled from concentric glass rods; (b) polymer-coating of glass seeds; (c) selective dissolution of high RI ( 1.76) alkali-Ba-borosilicate glasses; and (d) by "coating" glass spheres (1 to $2 \mathrm{~mm}$ diameter) with molten glass in a two step process.

2.1. Fiber pulling and Spheroidization. This method is based on multiple-coating of glass rods with other glasses of lower RI values. The method requires that all glasses have similar thermal expansion coefficient and melting temperatures. The "cladded" glass rods are then pulled into fibers of appropriate diameter ( 1 to $3 \mathrm{~mm}$ ), chopped in appropriate lengths ( 1 to $3 \mathrm{~mm}$ ), and spheroidized (Fig. 2). Although spheroidization of the chopped fibers produced non-concentric layers (Fig. 3), the method is simple enough for scaling up to produce many beads almost simultaneously, at relatively low cost, and could produce glass spheres with GRIN value for some applications.

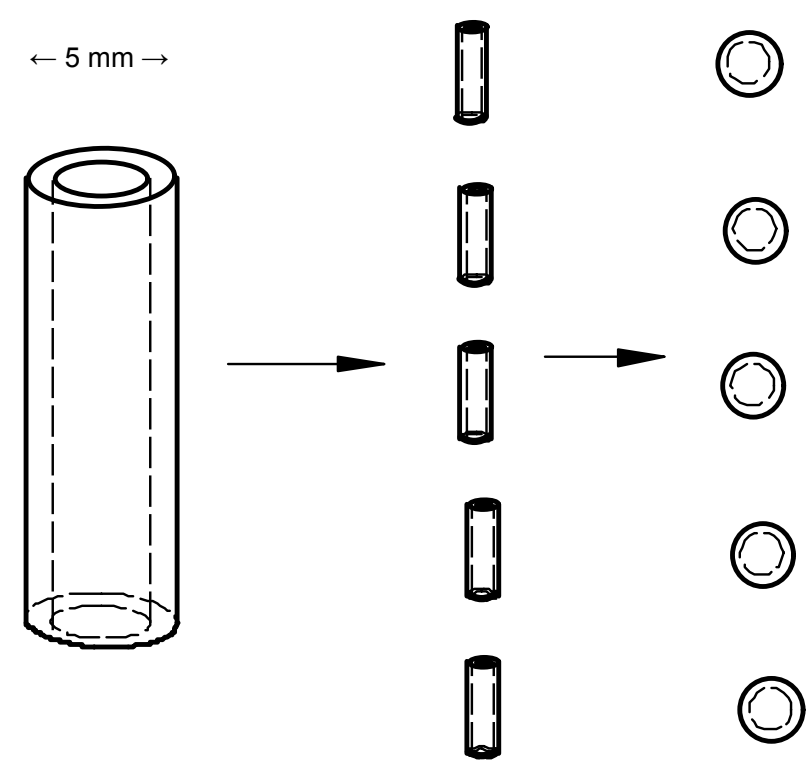

Figure 2. (a) Diagram illustrating the fabrication of GRIN glass spheres from a glasscladded glass-rod: fiber pulling, fiber chopping, and spheroidization. 

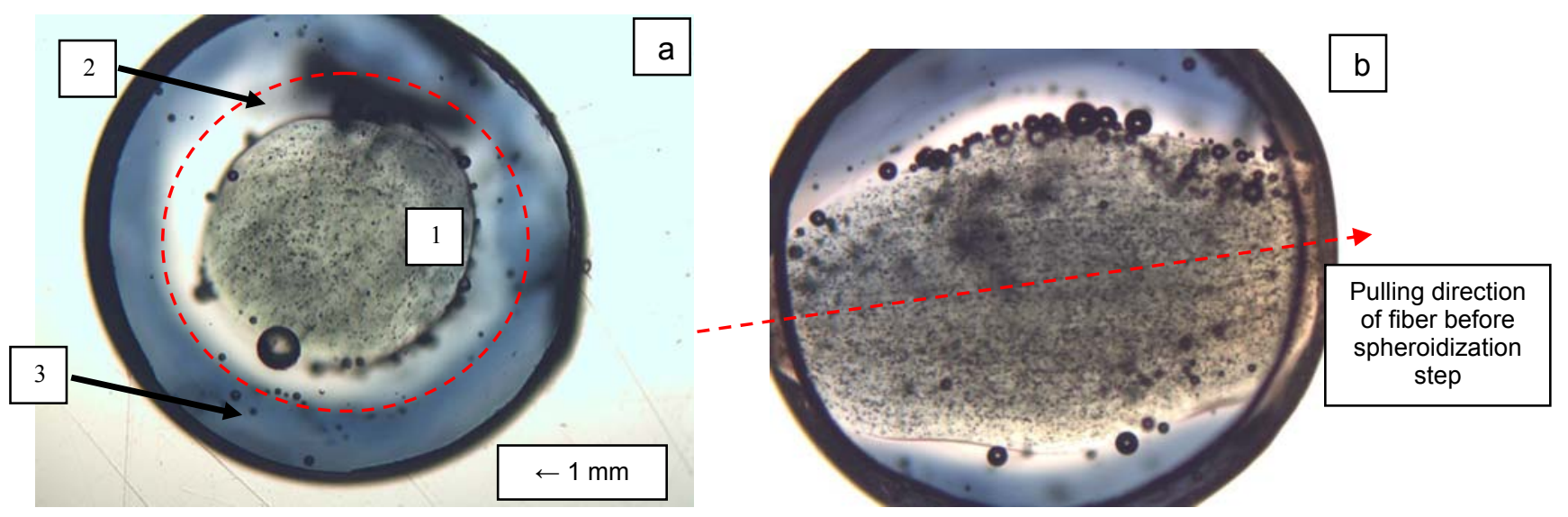

Figure 3. Cross section of a two-coating-layer simulated-GRIN glass sphere obtained by spheroidization of a cladded glass fiber. (a) The GRIN glass sphere consists of a central glass (labeled 1), and two glass layers (labeled 2 and 3). A dashed red circle is added to help visualize the two glass layers cladding the central glass. The GRIN glass sphere shows bubbles introduced during the process. The black specks in the core glass are due to metal frit that was added to track the "orientation" of the fiber pulling. (b) A dashed red line is added to help visualizing the core orientation of the fiber pulling.

2.2. Polymer Coating of Glass Seeds. The goal was to produce a $10 \mu \mathrm{m}$ layer of poly(methyl methacrylate) (PMMA) polymer around $1 \mathrm{~mm}$ glass spheres. The method used a series of monomers to be polymerized producing a series of uniform layers of varying $\mathrm{RI}$ according to the homopolymers refractive indices. The advantage of the process is that it is scalable to any size of reaction, bead size, or varying RI and allows for precisely tailoring the change in $\mathrm{RI}$ over the layer thickness. Additionally, glass seeds of high RI (i.e., 1.9) can be coated with sequential polymer layers at room temperature $\left(23^{\circ} \mathrm{C}\right)$, avoiding post-thermal heat treatment of the glass seed.

Atom transfer radical polymerization (ATRP -- polymerization process involving free radicals that allows the reaction to be carried out in a controlled way, and can be used to obtain polymers with high molecular weight and low polydispersity index) was chosen to show that a monomer could be polymerized in sufficiently thick layers [9-11]. Polymerization of PMMA around the glass spheres was performed after modifying the glass surface with a silane and 2,2,3 trimethyl-3-bromo-propionyl bromide [12].

The glass spheres were reacted for 3 days at $70^{\circ} \mathrm{C}$ under argon atmosphere. The reaction was quenched with air and the glass spheres rinsed with methanol, dried under vacuum overnight, mounted in epoxy-amine matrix, and polished to produce a cross section (SEM, Fig. 4). The PMMA layer thickness was observed to be at, or just less than, $1 \mu \mathrm{m}$. While the ATRP methodology was not successful at producing the 
desired layer thickness, it is believed that the process could be made with faster rates, able to produce block layers of a millimeter or more in thickness. Additionally, the 1-layer coated glass microspheres can be further treated with other monomers tailoring a sequential RI profile.
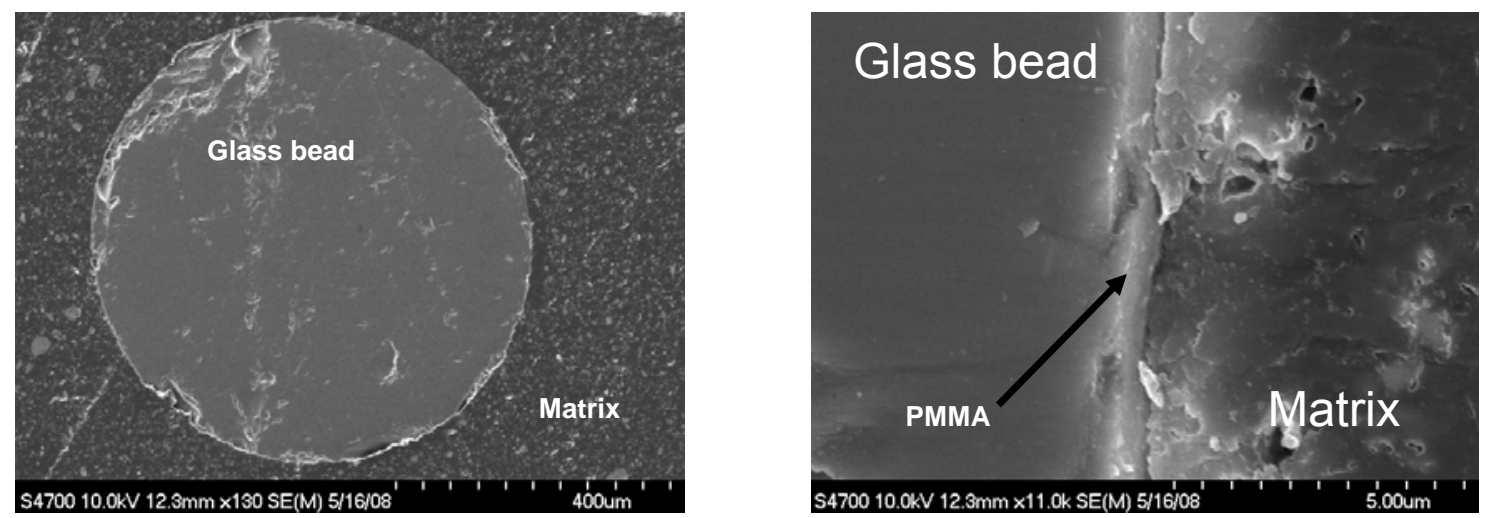

Figure 4. SEM image of glass sphere with PMMA surface layer. The PMMA interphase is estimated to be $0.5 \mu \mathrm{m}$ thick.

2.3. Selective Dissolution of Glass Spheres. In this process a high-RI alkali-Ba-Ticontaining borosilicate glass-sphere $(\mathrm{RI} 1.76)$ is placed in $\mathrm{HCl}$ solution where the $\mathrm{Ba}$ ions are leached out, leaving an outer glass-silica region of lower RI value. After leaching all $\mathrm{Ba}$ and $\mathrm{Ti}$ from a thick (300 to $400 \mu \mathrm{m}$ ) surface layer, the glass spheres were heat treated under several conditions (temperature and time) to determine feasibility of re-melting the spheres. Although the method shows potential for manufacturing only a 1layer GRIN glass sphere, the optical quality is low due to defects in the silica layer.

2.4. Molten Glass Coating. Glass spheres (ideally seeds of high RI), held with vacuum at the end of a tube, were dipped into a glass melt of lower RI (Fig. 5). The method is a two-dipping process, as the seeds are hemi-spherically coated in each step. The doublecoated glass seeds were then passed through a hot drop tube furnace to re-melt the glass surface and homogenize the glass coating. A blue-colored glass (Li-B-Co-silicate glass), was used as molten glass while two types of glass seeds were used for testing the concept:

- $\quad$ Li-Ba-borosilicate glass spheres, $\sim 2$ mm diameter, RI 1.624; and

- Commercial Na-silicate glass spheres, $\sim 2$ mm diameter, RI 1.52.

The two Li-borosilicate glasses (blue-colored glass and Li-Ba-borosilicate glass spheres) were designed to have low RI (1.558) and high RI (1.624), respectively, while having chemical compatibility and similar coefficient of thermal expansion. The commercial Na-silicate glass spheres were chosen for availability and to test the effect of using glass seeds with different CTE values compared to the intended glass coating. 


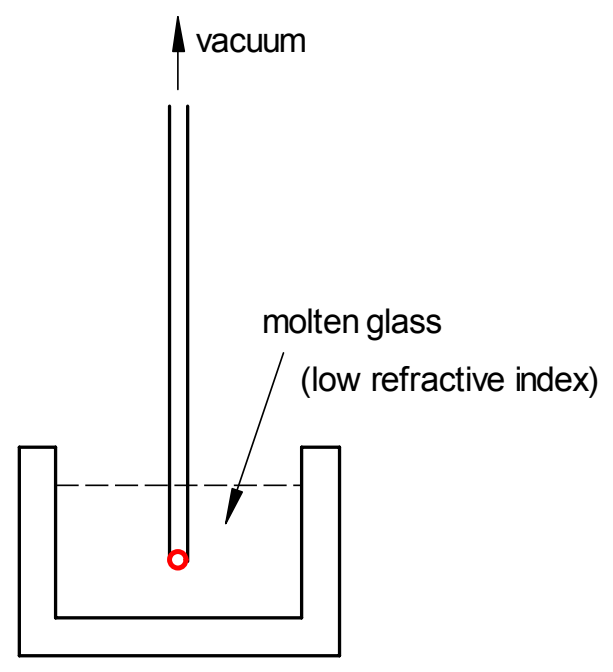

Figure 5. Schematics for coating a hemi-sphere glass seed (of high refractive index) with molten glass (of low refractive index). The glass seed is held by vacuum while is immersed in an amount of molten glass.

Figure 6 shows a coated hemi-sphere of a Li-Ba-silicate glass seed, showing a uniform coating. Figure 7 shows the polished cross-section of same glass seed (Fig. 6) after a two step coating (complete coating) and the interface between this glass coating and the glass seed. A very thick (100 to 300 microns) and concentric coating was made with a sharp and defect-free interface, which is needed for optical quality.

Figure 8 shows the polished cross-section of a coated Na-silicate glass seed and the detail of the sharp interface between the blue glass coating and the Na-silicate seed. A 200 to 300 microns thick and concentric coating was obtained. However, the glass coating has cracks due to different coefficient of thermal expansion between the coating and the glass seed. This proves that the glass coating and the glass seed need to have similar CTE values to avoid thermal defects.

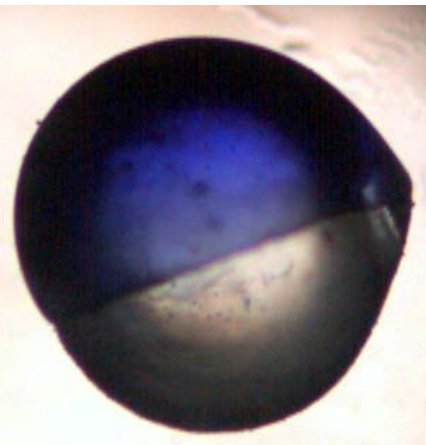

Figure 6. Coated hemi-sphere of a Li-Ba-silicate glass seed. The two-step coating process produces almost uniformly coated seeds, as shown in Fig. 7 and 8. 

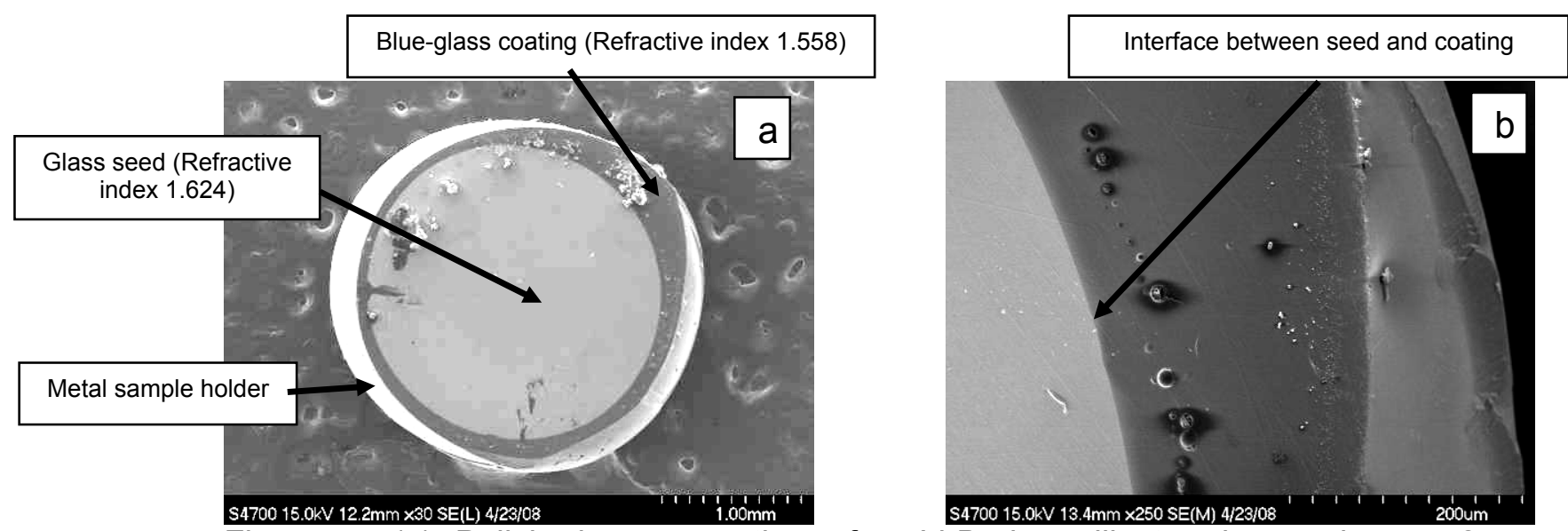

Figure 7. (a) Polished cross-section of a Li-Ba-borosilicate glass sphere, $\sim 2 \mathrm{~mm}$ diameter, refractive index 1.624, after being coated with a Li-B-Co-silicate glass (refractive index 1.558) of similar coefficient of thermal expansion. The coating is concentric and very thick, 100 to 300 microns; (b) detail of the interface between the blue-glass coating and the glass seed.
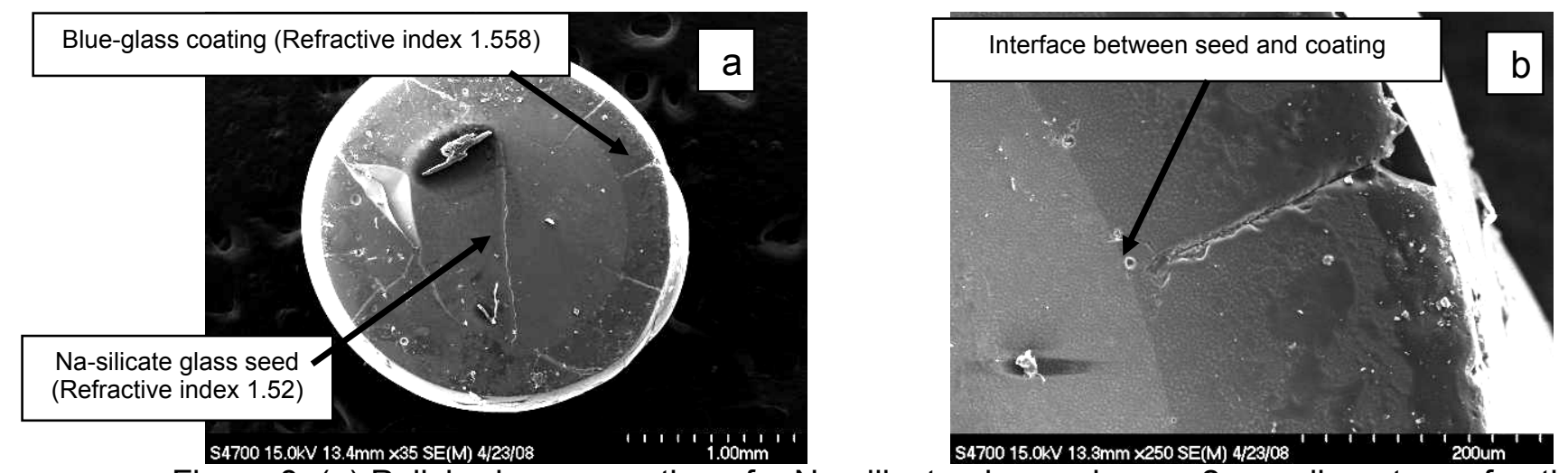

Figure 8. (a) Polished cross-section of a Na-silicate glass sphere, $\sim 2 \mathrm{~mm}$ diameter, refractive index 1.52, after being coated with a Li-B-Co-silicate glass (refractive index 1.558) of lower coefficient of thermal expansion. The coating is concentric and very thick, 200 to 300 microns but shows cracks due to thermal stresses; (b) detail of the sharp interface between the blueglass coating and the glass seed.

\section{CONCLUSIONS}

The recommended ways to make the GRIN spheres are (1) by "coating" glass spheres ( 1 to $2 \mathrm{~mm}$ diameter) with molten glass in a two step process; and (2) by coating glass spheres with polymer layers. The results indicate that 1 to $3-\mathrm{mm}$ diameter glass spheres can be coated with at least one concentric glass layer of thickness between 100 and 300 microns. The "molten glass coating" process can be refined by tailoring the glass seed composition and the glass coating composition regarding refractive index and the coefficient of thermal expansion. 


\section{REFERENCES}

1. N.C. Anheier, B.E. Bernacki, K. Krishnaswami, Miniature Spherical Retroreflectors, Technical Report PNNL-16320, December 2007.

2. P.C. Kapur, D.W. Fuerstenau, Size Distribution and Kinetic Relationships in the Nuclei Region of Wet Pelletization, I\&EC Process Design and Rev., 5[1] 5-10 (1966).

3. H.P. Meissner, A.S. Michaels, R. Kaiser, Rate of Pelletization of Zinc Oxide, I\&EC Process Design and Rev., 5[1] 10-14, (1966).

4. H.A. Rashid, Centrifugal Granulating Process for Preparing Drug-Layered Pellets Based on Microcrystalline Cellulose, Thesis Pharmacy Dept., University of Helsinki, 2001.

5. J.J. Hagerty, D.N. Pulsifer, Glass Plate Fusion for Macro-Gradient Refractive Index Materials, U.S. Patent 4,929,065, May 1990, assigned to ISOTEC Partners, Ltd.

6. X. Xu, M.E. Savard, Process for Manufacturing GRIN Lenses by Melting a Series of Layers of Frit, U.S. Patent 5,630,857, May 1997, assigned to LightPath Technologies, Ltd.

7. S. Jiang, P. Lam, Method for fabricating Gradient-Index Rods and Rod Arrays, U.S. Patent 6,598,429, Jul. 2003, assigned to Beam Tek, Inc.

8. http://www.us.schott.com/optics_devices/english/download/minikatalog_us.pdf

9. K. Matyjaszewski, J.H. Zia, Atom Transfer Radical Polymerization, Chem. Rev., 101, 2921-2990 (2001);

10. M. Biesalski, J. Ruhe, Swelling of a Polyelectrolyte Brush in Humid Air, Langmuir, 16, 1943-1950 (2000).

11. Q. Zhou, X. Fan, C. Xia, J. Mays, R. Advincula, R., Living Anionic Polymerization of Styrene from Clay Surfaces, Polymeric Mat. Sci. and Eng., 84, 835-836 (2001).

12. Z. Bao, M.L. Bruening, G.L. Baker, G.L., Rapid Growth of Polymer Brushes from Immobilized Initiators, J. Am. Chem. Soc., 128, 9056 (2006) 